\title{
Identification of plasma microRNA-21 as a biomarker for early detection and chemosensitivity of non-small cell lung cancer
}

\author{
Juan Wei, Wen Gao, Cheng-Jun Zhu, Yi-Qian Liu, Zhu Mei, Ting Cheng and Yong-Qian Shu
}

\begin{abstract}
Studies have shown cell-free microRNA (miRNA) circulating in the serum and plasma with specific expression in cancer, indicating the potential of using miRNAs as biomarkers for cancer diagnosis and therapy. This study was to investigate whether plasma miRNA-21 (miR-21) can be used as a biomarker for the early detection of non-small cell lung cancer (NSCLC) and to explore its association with clinicopathologic features and sensitivity to platinum-based chemotherapy. We used real-time RT-PCR to investigate the expression of miR-21 in the plasma of 63 NSCLC patients and 30 healthy controls and correlated the findings with early diagnosis, pathologic parameters, and treatment. Thirty-five patients (stages IIIB and IV) were evaluable for chemotherapeutic responses: 11 had partial response (PR); 24 had stable and progressive disease (SD+PD). Plasma miR-21 was significantly higher in NSCLC patients than in age- and sex-matched controls $(P<0.001)$. miR-21 was related to TNM stage $(P<0.001)$, but not related to age, sex, smoking status, histological classification, lymph node status, and metastasis (all $P>0.05)$. This marker yielded a receiver operating characteristic $(\mathrm{ROC})$ curve area of $0.775 \quad(95 \% \mathrm{Cl}$ : $0.681-0.868)$ with $76.2 \%$ sensitivity and $70.0 \%$ specificity. Importantly, miR-21 plasma levels in PR samples were several folds lower than that in SD plus PD samples $(P=0.049)$, and were close to that in healthy controls $(P=0.130)$. Plasma miR-21 can serve as a circulating tumor biomarker for the early diagnosis of NSCLC and is related to the sensitivity to platinum-base chemotherapy.
\end{abstract}

Key words Lung neoplasm, non-small cell, miRNA-21, biomarker, plasma, early detection, chemosensitivity

Lung cancer, one of the most common malignant tumors, is a serious disease threatening human life and health. Of the pathologic types of lung cancer, non-small cell lung cancer (NSCLC) is the most prevalent (80\%). The lack of effective early diagnostic and treatment methods are the main causes of high mortality in patients with lung cancer, with the 5-year survival rate at $15 \%$ to $20 \%$. MicroRNA (miRNA) is a class of 20 to 25 nt long non-coding RNA found in vivo. miRNA genes are transcribed in the nucleus to primary transcripts

\footnotetext{
Authors' Affiliation: Department of Oncology, The First Affiliated Hospital, Nanjing Medical University, Nanjing, Jiangsu 210029, P. R. China.

Corresponding Author: Yong-Qian Shu, Department of Oncology, The First Affiliated Hospital, Nanjing Medical University, Nanjing, Jiangsu 210029, P. R. China. Tel: +86-25-68136428; Fax: +86-25-83710040; Email: shuyongqian@csco.org.cn.
}

(pri-miRNA) ${ }^{[1]}$. After digestion by Drosha, precursor miRNA (pre-miRNA) is then formed and is transported to the cytoplasm and digested by Dicer to produce mature miRNA ${ }^{[2,3]}$, which regulates gene expression mainly through its partially complementary to $3^{\prime}$-untranslated region ( $3^{\prime}$ UTR) of target $m R N A^{[4]}$.

Among their functional roles, miRNAs regulate development, organogenesis, hematopoiesis, cell proliferation and apoptosis, and tumorigenesis and progression. It has been confirmed that miRNAs are involved in the development of a variety of tumors, such as leukemia ${ }^{[5]}$, neuroblastoma ${ }^{[6]}$, pituitary adenoma ${ }^{[7]}$, breast cancer ${ }^{[8]}$, thyroid cancer ${ }^{[9]}$, hepatocarcinoma ${ }^{[10]}$, colorectal cancer ${ }^{[11]}$, and lung cancer ${ }^{[12]}$. The up- or down-regulation of different miRNAs in these tumor tissues were found with most of the miRNA targets located in regions of tumor-related genome, fragile sites, loss of heterozygosity, and amplified regions ${ }^{[5]}$, thereby exhibiting the same effect as oncogenes or tumor 
suppressor genes.

An increasing number of studies have shown that analysis of miRNA expression has a potential application value in tumor diagnosis, treatment, and prognosis prediction. It has been observed that miRNAs are closely related to tumor development and stably exist in serum/plasma ${ }^{[13-15]}$. The origin of miRNAs is unclear and lacks experimental evidence, but current assumptions of the origin include cancer cells in peripheral blood ${ }^{[16]}$ or tumor-associated bodies ${ }^{[17,18]}$. The first miRNA marker found in serum is miRNA-21 (miR-21). Lawrie et al. ${ }^{[19]}$ proved that high expression of miR-15, miR-21, and miR-210 existed in the serum of patients with diffuse large B-cell lymphoma (DLBCL) using real-time fluorescence quantitative PCR, and miR-21 with largest differential expression is related to lymphoma recurrence and patient survival. Subsequently, Mitchell et al. ${ }^{[16]}$ found that miR-141 was highly expressed in patients with metastatic prostate cancer. The level of miR-141 expression is highly specific and sensitive for the diagnosis of prostate cancer and thus, can serve as a marker. In addition, the level of miR-184 expression in the plasma of patients with tongue squamous cell carcinoma was significantly higher than that in healthy people and was decreased significantly after tumor removal and corresponding treatment ${ }^{[20]}$. $\mathrm{Ng}$ et al. ${ }^{[21]}$ found that the expression levels of miR-17-3p and miR-92 in the plasma and tissue of patients with rectal cancer were significantly increased and that the expression of miR-92 was significantly reduced after tumor resection, suggesting that miR-92 can function as a serum molecular marker for the diagnosis of colorectal cancer at stage I-IV, whereas early diagnosis can improve survival and prognosis. The expression of miR-155 in patients with progesterone receptor-positive breast cancer was higher than that in progesterone receptor-negative patients, suggesting that miR-155 can serve as a molecular marker differentiating hormonesensitive breast cancer from non-sensitive ones ${ }^{[2]}$. These studies indicate that plasma/serum miRNAs may function as potential biomarkers for the non-invasive diagnosis, treatment, and prognosis of cancers.

In the present study, we measured the plasma level of miR-21 and determined its relationship to the early diagnosis of NSCLC, efficacy of chemotherapy, and clinicopathologic characteristics of patients with NSCLC.

\section{Materials and Methods}

\section{Study subjects}

Peripheral blood specimens from 63 patients with pathologically confirmed NSCLC were collected between October 2009 and August 2010 from the Departments of
Thoracic Surgery and Oncology in the First Affiliated Hospital of Nanjing Medical University. Of the 63 patients, 45 were men and 18 were women, aged of 36 to 83 years with a median of 61 years. The patients did not undergo chemotherapy or radiotherapy prior to admission. Venous blood was drawn before surgery for patients with early stage NSCLC. For those with advanced NSCLC, CT was used to confirm measurable tumor lesions. In addition, functional scores before chemotherapy were greater than 60 , with blood and kidney function in the normal range and a normal electrocardiogram. Venous blood was drawn before chemotherapy for all patients. According to the 7th edition of TNM staging of the Union for International Cancer Control (UICC) and American Joint Committee on Cancer (AJCC) in 2009, 18 cases were classified as stage I, 5 as stage II, 5 as stage III, and 35 as stage IV. Thirty-eight patients had adenocarcinoma, 15 had squamous cell carcinoma, 1 had large cell carcinoma, and 9 had unclassified tumors. In addition, 30 had lymph node metastasis and 33 had no lymph node metastasis. The control group was composed of 30 healthy people: 20 men and 10 women aged 41 to 75 years with a median of 57 years. There was no statistical difference in age $(P$ $=0.086$, Mann-Whitney test $)$ and sex $\left(P=0.803, \chi^{2}\right.$ test) between NSCLC patients and the control group.

\section{Chemotherapy regimen and the evaluation of therapeutic efficacy}

Of the 63 patients, 40 underwent cisplatin- or carboplatin-based chemotherapy; 35 had measurable lesions. The chemotherapy regimen was repeated every 3 to 4 weeks. After 2 to 3 cycles of treatment, an objective evaluation was made according to the standards of Evaluation Criteria in Solid Tumors recommended by the WHO, defined as complete remission (CR), partial remission (PR), stable disease (SD), and progressive disease (PD).

\section{Real-time quantitative RT-PCR detection of miR-21 expression in plasma}

Peripheral blood specimen $(5 \mathrm{~mL})$ was conventionally drawn from each patient before surgery or chemotherapy. EDTA was used as an anticoagulant. Plasma and blood cells were separated by centrifugation at $1200 \times g$ for $10 \mathrm{~min}$ at $4^{\circ} \mathrm{C}$, and stored in $1.5 \mathrm{~mL}$ RNase-free tubes at $-80^{\circ} \mathrm{C}$. Peripheral blood was processed within $4 \mathrm{~h}$.

Plasma $(400 \mu \mathrm{L})$ from each sample was centrifuged at $1200 \times g$ for $10 \mathrm{~min}$ at $4^{\circ} \mathrm{C}$ before RNA extraction. Total RNA was extracted using mirVana PARIS kit according to manufacturer's instruction (Ambion, USA). To eliminate differences among samples, synthetic 
Caenorhabditis elegans cel-miR-39, which has no homologous gene in human, was added into each sample. RNA was dissolved in $100 \mu \mathrm{L}$ of RNase-free water. Absorbance at 260 and $280 \mathrm{~nm}$ was measured using a spectrophotometer to determine the concentration and purity of RNA.

The expression of miR-21 in NSCLC patients and healthy controls was detected by using miScript SYBR Green PCR kit (Qiagen, Germany). The reaction was performed in a real-time quantitative $P C R$ machine $\mathrm{ABI} 7300 \mathrm{HT}$. Reverse transcription of miRNA was performed according to manufacturer's instruction using the miScript Reverse Transcription kit (Qiagen). The first chain of cDNA was reverse transcribed from $2 \mu \mathrm{L}$ of total RNA using specific stem-loop structure RT primers of miR-21. The amount of RNA was determined by the volume of plasma: $(2.0 / 80) \times 400=10 \mathrm{~mL}, 2 \mu \mathrm{L}$ of RNA corresponding to $10 \mu \mathrm{L}$ plasma ${ }^{[16]}$. The reverse transcription reaction was carried out at $37^{\circ} \mathrm{C}$ for $60 \mathrm{~min}$ and at $95^{\circ} \mathrm{C}$ for $5 \mathrm{~min}$. Subsequently, PCR was performed using the first chain of CDNA as the template. The reaction system contained $2 \mu \mathrm{L}$ of $\mathrm{cDNA}, 12.5 \mu \mathrm{L}$ of $1 \times$ SYBR Green I Master mix, $2.5 \mu \mathrm{L}$ of miRNA specific primers, and $2.5 \mu \mathrm{L}$ of upstream universal primer. The reaction conditions were as follows: denatured at $95^{\circ} \mathrm{C}$ for $15 \mathrm{~min}, 40$ cycles of $95^{\circ} \mathrm{C}$ for $15 \mathrm{~s}, 57^{\circ} \mathrm{C}$ for $30 \mathrm{~s}$, and $70^{\circ} \mathrm{C}$ for $34 \mathrm{~s}$. miR-16 was used as an internal reference for normalization. An average of triplicate findings was considered as the result for each sample. The sequence of cel-miR-39 was 5'-UCACCGGGUGUAAAUCAGCUUG-3'; the sequence of miR-16 primer was 5'-UAGCAGCACGUAAAUAUUGGCG-3'; the sequence of miR-21 primer was 5'-UAGCUUAUCAGACUGAUGUUGA-3' (synthesized by Qiagen). Finally, Ct values were measured using melting curve analysis to represent the expression of target gene mRNA. Relative changes of gene expression were represented by $2-\Delta \Delta \mathrm{C}$, the difference between the original copy number of miR-21 in the NSCLC group and that in the normal group. $\triangle \triangle \mathrm{Ct}=\left(\mathrm{Ct}_{\text {miR-21 }}-\mathrm{Ct}_{\text {miR-16 }}\right)$ of the NSCLC group$\left(\mathrm{Ct}_{\text {miR-21 }}-\mathrm{Ct}_{\text {miR-16 }}\right)_{\text {mean }}$ of the control group.

\section{Statistical analysis}

GraphPad Prism V4.03 and SPSS16.0 statistical softwares were used for statistical analysis. Log10 normality conversion was used to analyze the relative expression of miR-21. The Mann-Whitney $U$ test was used for data comparison between two groups. The Kruskall-Wallis $H$ test was used for comparison among multiple groups. The receiver operating characteristic (ROC) curve was drawn to evaluate the diagnosis value of plasma miR-21 for NSCLC. $P<0.05$ indicates a statistical significance.

\section{Results}

The expression levels of plasma miR-21 in NSCLC group and healthy control group detected by real-time quantitative RT-PCR

Using miR-16 as an internal reference, the expression level of plasma miR-21 was detected by real-time fluorescence quantitative PCR. The quantitative PCR amplification and melting curves reveal the expression levels of miR-21 and miR-16 (Figure 1).

\section{Relationship between plasma miR-21 expression and clinicopathologic features of NSCLC}

PCR results showed that miR-21 expression was significantly higher in the NSCLC group than in the control group $(P<0.001)$ (Figure 2). miR-21 expression was not related to age, sex, smoking status, pathologic type, lymph node metastasis, and distant metastasis (all $P>0.05)$, but related to TNM stage $(P<0.001)$. Plasma miR-21 expression was higher in stage T3-4 patients than in stage T1-2 patients $(P=0.036)$ and was higher in stage III-IV patients than in stage I-II patients $(P=$ 0.043) (Figure 2), indicating that plasma miR-21 is important for the diagnosis of stage I-IV NSCLC. The area under the ROC curve (AUC) for plasma miR-21 was $0.775(95 \% \mathrm{Cl}: 0.681-0.868)$, the sensitivity and specificity were $76.2 \%$ and $70.0 \%$, respectively, and the value of the cut-off point was 1.308 (Figure 3 ).

\section{Relationship between plasma miR-21 expression and chemotherapeutic efficacy}

Thirty-five patients with advanced NSCLC who underwent 2 to 3 cycles of platinum-base chemotherapy were followed up: 11 had PR, 13 had PD, 11 had SD, and none achieved CR after chemotherapy. The expression of plasma miR-21 was significantly higher in the PD plus SD group than in the PR group $(P=0.049)$, whereas no statistical significance was found between the PR group and the control group $(P=0.130)$ (Figure 4 ), suggesting that plasma miR-21 expression may correlate with sensitivity to chemotherapy in NSCLC.

\section{Discussion}

miR-21 is overexpressed in lung cancer, breast cancer, glioblastoma, gastric cancer, pancreatic cancer, pancreatic neuroendocrine tumor, liver cancer, bile duct cancer, colon cancer, and prostate cancer ${ }^{[23]}$. miR-21 is encoded by a single gene, which locates at the fragile site FRA17B on 17p23.2 and is overlapped with the 


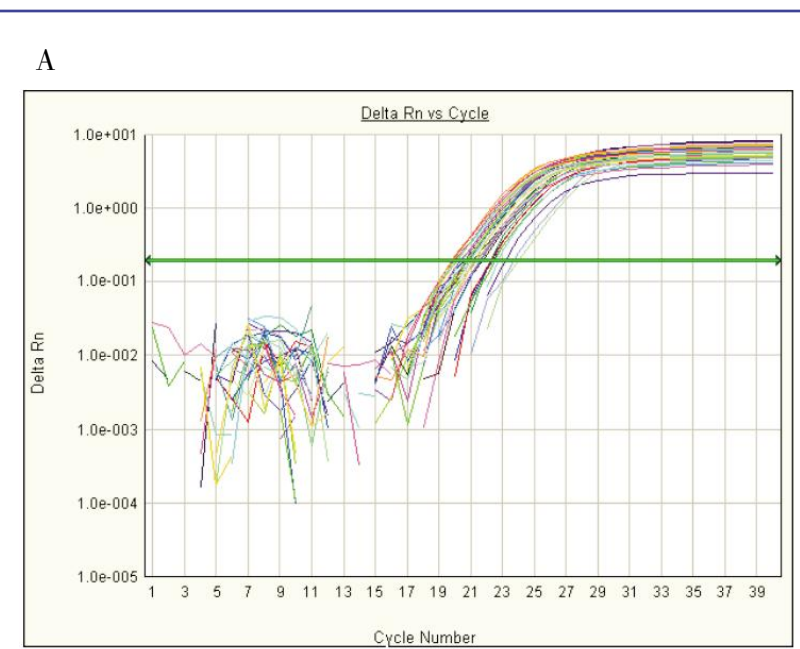

C

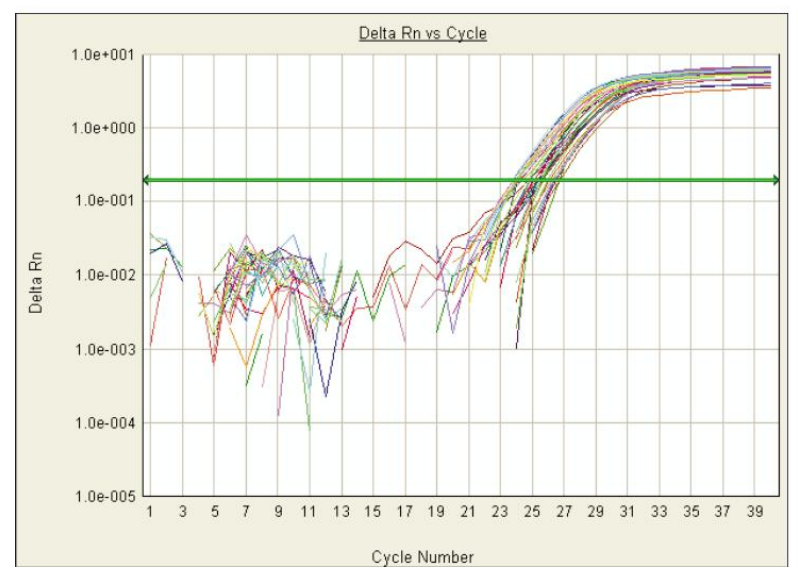

B

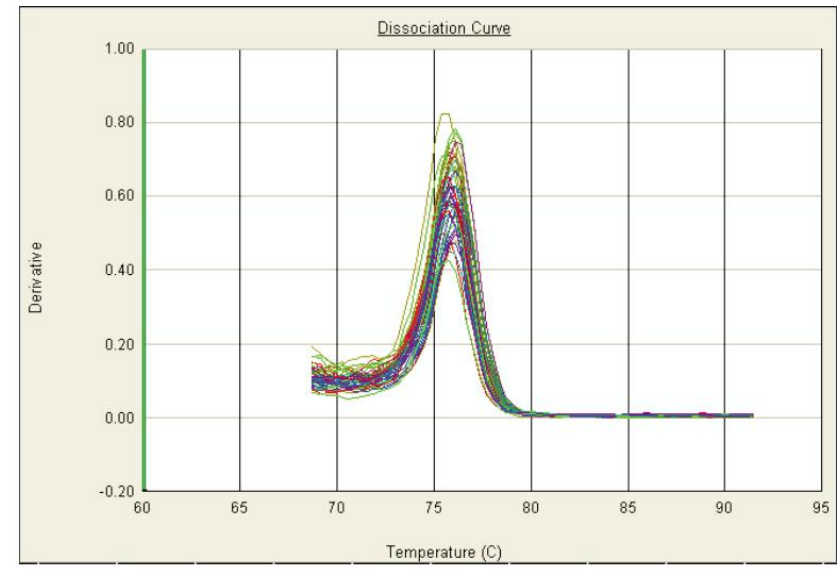

$\mathrm{D}$

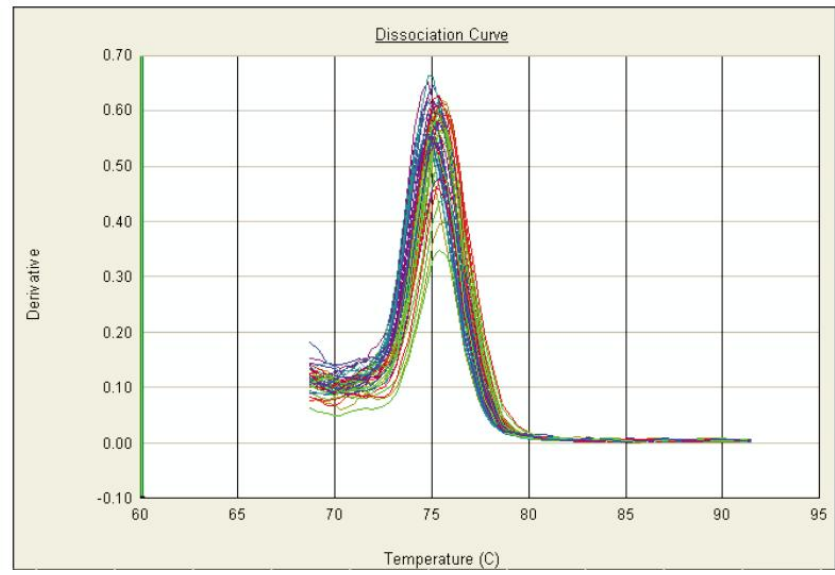

Figure 1. The amplification and melting curves of miR-16 and miR-21. A, amplification curve of miR-16; B, melting curve of miR-16; C, amplification curve of miR-21; D, melting curve of miR-21. Quantitative fluorescence amplification curves show the relationship between fluorescence and the number of cycles. The cycle threshold (Ct) is defined as the number of cycles required for the fluorescent signal to cross the threshold. Ct levels are inversely proportionate to the amount of target nucleic acid in the sample: the lower the Ct level, the greater the amount of target nucleic acid in the sample. Ct $\leqslant 29$ indicates strong positive reactions indicative of abundant target nucleic acid in the sample. Melting curve is the quality control of amplification. It is used to analyze the homogeneity of PCR products. The results show that the Ct value of miR-16 is about 21 , the Ct value of miR-21 is about 25 , and the $\mathrm{Tm}$ is about $75^{\circ} \mathrm{C}$. Impure or abnormal broaden peaks do not appear in the figure, indicating, that there is no contamination, primer dimmers, or nonspecific amplification.

gene encoding transmembrane protein 49 (TMEM49, also known as VMP-1 $)^{[24]}$. miR-21 contains 22 nucleotides and is separated from a 3400 nt pre-transcript ${ }^{[25]}$. Abnormal expression of miR-21 may be related to transcription and post-transcriptional regulation ${ }^{[24]}$. miR-21 regulates cell differentiation, proliferation, and apoptosis and is involved in tumor cell invasion, vascular infiltration, and metastasis by regulating target genes, including tropomyosin 1 (TPM1), programmed cell death gene 4 (PDCD4), methyl-adenosine phosphatase (MTAP), SOX5, genes in the p53-mediated pathway and transforming growth factor (TGF- $\beta$ ) pathway, Bcl-2, phosphatase and tensin homolog (PTEN). In addition, the high expression of miR-21 in patients with colorectal cancer is correlated to clinical stage, lymph node metastasis, and distant metastasis ${ }^{[26]}$; stages of breast cancer and liver metastases accompany with the increasing expression level of miR-2 $1^{[8]}$. Several studies have also confirmed that miR-21 plays an important clinical role in tumor diagnosis and evaluation. Schetter et al. ${ }^{[27]}$ found that high expression of miR-21 in colon cancer is related to poor efficacy of treatment and prognosis. Yan et al. ${ }^{[28]}$ found that overexpression of miR-21 in breast cancer is associated to lymph node 


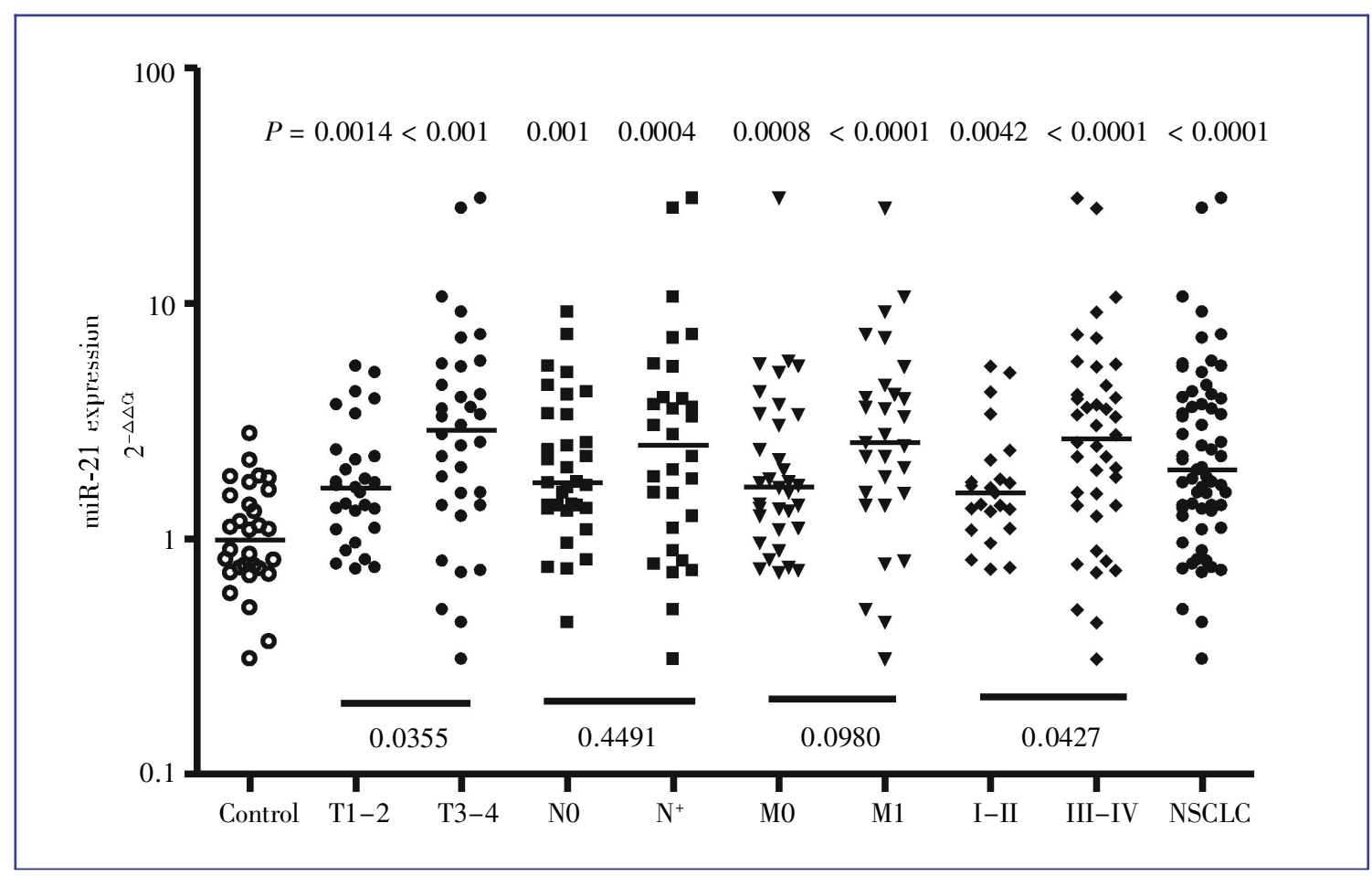

Figure 2. The correlation between the expression of miR-21 and clinical parameters of non-small cell lung cancer (NSCLC) analyzed by MannWhitney $U$ test. The expression of miR-21 is significantly higher in NSCLC than in normal control $(P=0.001)$, higher in stage T3-4 NSCLC than in stage T1-2 NSCLC $(P=0.036)$, and higher in stage III-IV NSCLC than in stage I-II NSCLC $(P=0.043)$.

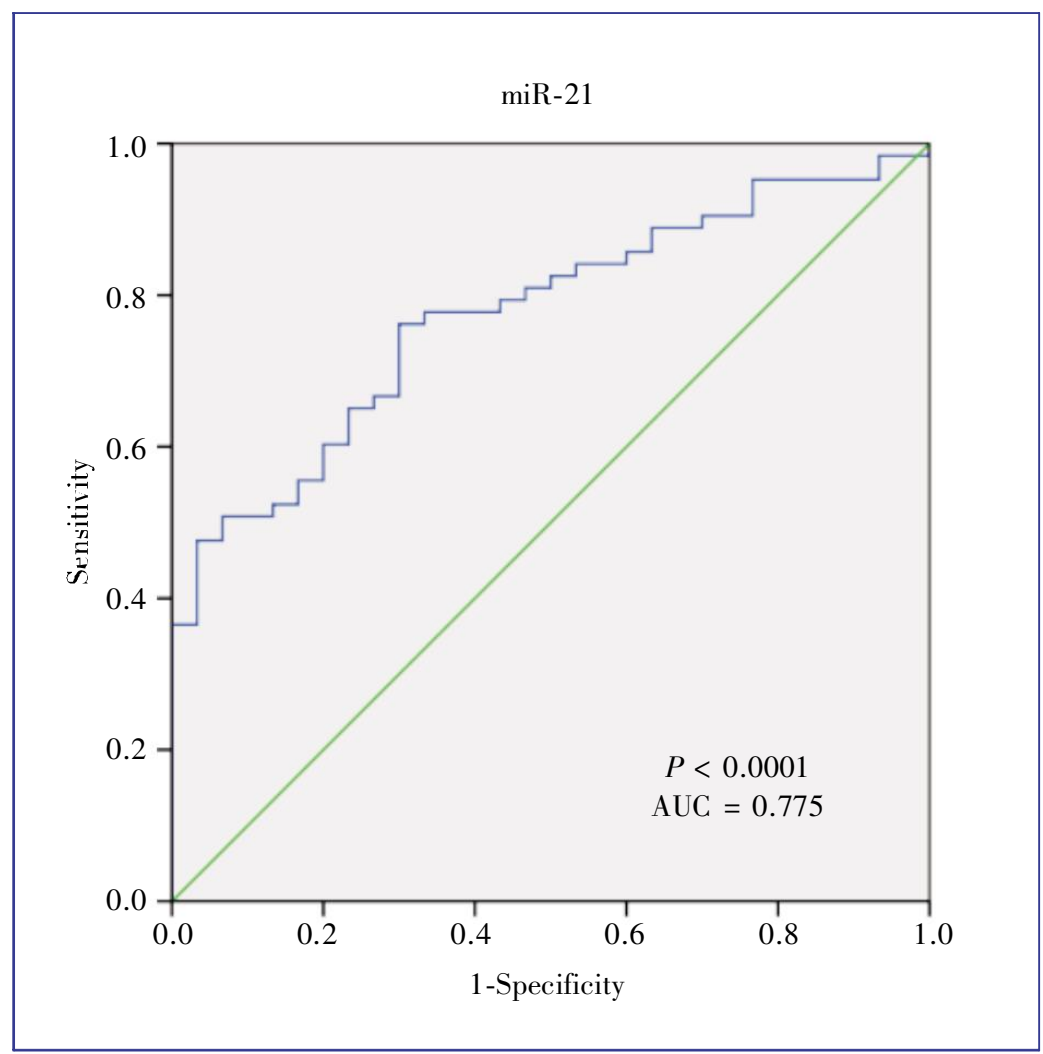

Figure 3. Diagnostic value of plasma miR-21 for NSCLC analyzed with receiver operating characteristics (ROC) curve. Plasma miR-21 yielded an (area under the ROC curve (AUC) of 0.775 (95\% Cl: $0.681-0.868)$ with $76.2 \%$ sensitivity and $70.0 \%$ specificity. The cut-off value was 1.308 . 


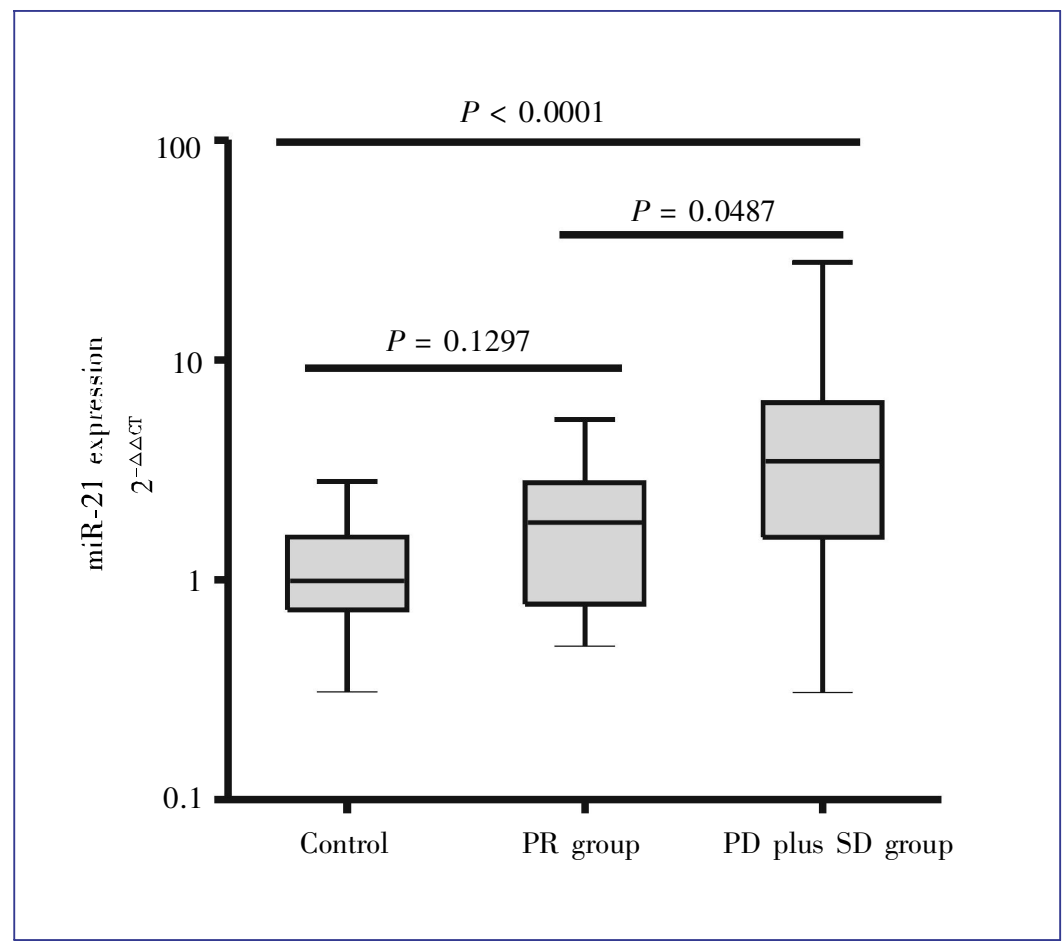

Figure 4. Plasma level of $m i R-21$ in patients with partial remission (PR) or progressive disease (PD) and stable disease (SD). Expression levels of the miR-21 (Log10 scale at $Y$-axis) are normalized to miR-16. Statistically significant differences were determined using the Mann-Whitney $U$ test. Plasma level of miR-21 is significantly higher in $\mathrm{PD}$ plus SD samples than in PR samples $(P<0.05)$ and healthy control samples $(P<0.001)$, and is similar between PR samples and control samples. The quantity of miR-21 in plasma may be a potential biomarker for predicting platinum-based chemo-sensitivity of NSCLC.

metastasis and poor prognosis. Gao et al. ${ }^{[29]}$ revealed that high expression of miR-21 in lung squamous cell carcinoma indicates poor prognosis. However, it has been reported that miR-21 only serves as a diagnostic marker in gastric cancer with no correlation to prognosis $^{[23]}$.

In this study, we used real-time quantitative PCR to determine the expression of plasma miR-21 in patients with NSCLC. Plasma miR-21 expression was significantly higher in NSCLC patients than in the control group $(P<0.0001)$. ROC curve analysis showed the AUC was 0.775 ; the sensitivity and specificity were $76.19 \%$ and $70.0 \%$. miR-21 expression was significantly related to TNM stage. The expression of plasma miR-21 was higher in patients with advanced NSCLC than in those with early stage NSCLC, suggesting that miR-21 expression may be related to factors associated with tumor progression. In particular, the expression of plasma miR-21 was significantly higher in patients with stage T1-2, N0 or I-II NSCLC than in the control group, suggesting that plasma miR-21 can be used as an effective, non-invasive marker for early NSCLC diagnosis.

Recently, studies have shown that miRNA-based treatment has a potential effect. Meng et al. ${ }^{[30]}$ found that the application of anti-miR-21 AMOs (anti-miRNA oligonucleotides) increase sensitivity of cholangiocarcinoma cells to gemcitabine. Si et al. ${ }^{[31]}$ treated miR-21/AMOs-transfected cancer cells with topotecan and found that antisense inhibition of
miR-21-mediated cell growth was significantly inhibited. $\mathrm{Li}$ et al. ${ }^{[32]}$ found that overexpression of miR-21 in malignant glioma cells leads to tumor cell resistance to chemotherapeutic drugs and that inhibiting the expression of miR-21 in malignant glioma U373MG cells by specific AMOs increases the sensitivity of U373MG cells $\mathrm{Vm}-26$, indicating that $\mathrm{miR}-21$ can be used to increase efficacy of drugs on malignant glioma. Hwang et al. ${ }^{[3]}$ retrospectively studied the effect of miR-21 on the chemotherapy efficacy and prognosis of pancreatic cancer: patients with low expression of miR-21 have significantly prolonged overall and disease-free survival after chemotherapy; antisense inhibition of miR-21 can enhance chemotherapeutic sensitivity. We followed up 35 patients with advanced NSCLC treated with platinum chemotherapy and found that the expression of plasma miR-21 in SD and PD patients was higher than that in PR patients. The expression of plasma miR-21 was low in $11 \mathrm{PR}$ patients, which showed no significant difference when compared to that in the control group. Thus, miR-21 may be related to platinum sensitivity. The high expression of plasma miR-21 may also be involved in cell resistance to platinum chemotherapy. Inhibiting miR-21 expression is likely to restore cell sensitivity to platinum chemotherapy. Therefore, our study provides a favorable basis to use miR-21 as a predictor of sensitivity to platinum chemotherapy. Due to a short follow-up duration, we cannot calculate the overall and disease-free survival, but we will continue to explore the relationship between plasma miR-21 expression and 


\section{NSCLC prognosis.}

In summary, our study demonstrated that the expression of plasma miR-21 is sensitive and specific for the early diagnosis of NSCLC and thus can be used as a potential biomarker for NSCLC diagnosis. Plasma miR-21 expression reflects sensitivity to platinum chemotherapy for NSCLC, suggesting that plasma miR-21 is a predictive indicator for sensitivity to platinum chemotherapy and may be a therapeutic target that reverse resistance to platinum chemotherapy.

\section{References}

[1] Lee Y, Ahn C, Han J, et al. The nuclear RNase III Drosha initiates microRNA processing [J]. Nature, 2003,425 (6956): $415-419$.

[2] Lund E, Guttinger S, Calado A, et al. Nuclear export microRNA precursors [J]. Science, 2004,303(5654):95-98

[3] Lee $\mathrm{Y}$, Jeon K, Lee JT, et al. MicroRNA maturation: stepwise processing and subcellular localization [J]. EMBO J, 2002,21 (17):4663-4670

[4] Rana TM. Illuminating the silence: understanding the structure and function of small RNAs [J]. Nat Rev Mol Cell Biol, 2007,8 (1):23-36

[5] Calin GA, Sevignani C, Dumitru CD, et al. Human microRNA genes are frequently located at fragile sites and genomic regions involved in cancers [J]. Proc Natl Acad Sci USA, 2004, 101(9): 2999-3004

[6] Chen $Y$, Stallings RL. Differential patterns of microRNA expression in neuroblastoma are correlated with prognosis, differentiation, and apoptosis [J]. Cancer Res, 2007,67(3):976983.

[7] Bottoni A, Piccin D, Tagliati F, et al. miR-15a and miR-16-1 down-regulation in pituitary adenomas [J]. J Cell Physiol, 2005, 204(1):280-285

[8] Iorio MV, Ferracin M, Liu CG, et al. MicroRNA gene expression deregulation in human breast cancer [J]. Cancer Res, 2005,65 (16):7065-7070.

[9] Jazdzewski K, Murray EL, Franssila K, et al. Common SNP in pre-miR-146a decreases mature miR expression and predisposes to papillary thyroid carcinoma [J]. Proc Natl Acad Sci USA, 2008, 105(20):7269-7274.

[10] Corney DC, Flesken-Nikitin A, Godwin AK, et al. MicroRNA-34b and microRNA-34c are targets of p53 and cooperate in control of cell proliferation and adhesion-independent growth [J]. Cancer Res, 2007,67(18):8433-8438.

[11] Lu J, Getz G, Miska EA, et al. MicroRNA expression profiles classify human cancers [J]. Nature, 2005,435(7043):834-838.

[12] Yanaihara N, Caplen N, Bowman E, et al. Unique microRNA molecular profiles in lung cancer diagnosis and prognosis [J]. Cancer Cell, 2006,9(3):189-198.

[13] Calin GA, Croce CM. MicroRNA signatures in human cancers [J]. Nat Rev Cancer, 2006,6(11):857-866

[14] Chen $X, B a Y, M a L$, et al. Characterization of microRNAs in serum: a novel class of biomarkers for diagnosis of cancer and other diseases [J]. Cell Res, 2008,18(10):997-1006.

[15] Filipowicz W, Bhattacharyya SN, Sonenberg N. Mechanisms of posttranscriptional regulation by microRNAs: are the answers in sight? [J]. Nat Rev Genet, 2008,9(2):102-114.

[16] Mitchell PS, Parkin RK, Kroh EM, et al. Circulating microRNAs

\section{Acknowledgements}

This work was supported by grants from the National Natural Science Foundation of China (No. 30772549) and the Medical Sci-Tech Development Foundation from Health Department of Jiangsu Province (No. P200965).

Received: 2010-11-05; revised: 2011-01-25; accepted: 2011-01-25. as stable blood-based markers for cancer detection [J]. Proc Natl Acad Sci USA, 2008,105(30):10513-10518.

[17] Resnike KE, Alder H, Hagan JP, et al. The detection of differentially expressed microRNAs from the serum of ovarian cancer patients using a novel realtime PCR platform [J] Gynecol Oncol, 2009,112(1):55-59.

[18] Taylor DD, Gercel-Taylor C. MicroRNA signatures of tumorderived exosomes as diagnostic biomarkers of ovarian cancer [J]. Gynecol Oncol, 2008,110(1):13-21.

[19] Lawrie CH, Gal S, Dunlop HM, et al. Detection of elevated levels of tumor-associated microRNAs in serum of patients with diffuse large B-cell lymphoma [J]. Br J Haematol, 2008,141(5): 672-675.

[20] Wong TS, Liu XB, Wong BY, et al. Mature miR-184 as potential oncogenic microRNA of squamous cell carcinoma of tongue [J]. Clin Cancer Res, 2008,14(9):2588-2592.

[21] Ng EK, Chong WW, Jin $\mathrm{H}$, et al. Differential expression of microRNAs in plasma of colorectal cancer patients: a potential marker for colorectal cancer screening [J]. Gut,2009,58(10): 1375-1381.

[22] Zhu W, Qin W, Atasoy $U$, et al. Circulating microRNAs in breast cancer and healthy subjects [J]. BMC Res Notes, 2009, 2:89.

[23] Chan SH, Wu CW, Li AF, et al. miR-21 microRNA expression in human gastric carcinomas and its clinical association [J] Anticancer Res, 2008,28(2A):907-911.

[24] Krichevsky AM, Gabriely G. miR-2l: a small multi-faceted RNA [J]. J Cell Mol Med, 2009,13(1):39-53.

[25] Cai X, Hagedorn $\mathrm{CH}$, Cullen BR. Human microRNAs are processed from capped polyadenylated transcripts that Call also function as mRNAs [J]. RNA, 2004,10(12):1957-1966.

[26] Slaby O, Svoboda M, Fabian P, et al. Altered expression of miR-21, miR-31, miR-143 and miR-145 is related to clinicopathologic features of colorectal cancer [J]. Oncology, 2007,72(5-6):397-402.

[27] Schetter AJ, Leung SY, Sohn JJ, et al. MiRNA expression profiles associated with prognosis and therapeutic outcome in colon adenocarcinoma [J]. JAMA, 2008,299(4):425-436.

[28] Yan LX, Huang XF, Shao Q, et al. MicroRNA miR-21 overexpression in human breast cancer is associated with advanced clinical stage, lymph node metastasis and patient poor prognosis [J]. RNA, 2008,14(11):2348-2360.

[29] Gao W, Shen H, Liu LX, et al. MiR-21 overexpression in human primary squamous cell lung carcinoma is associated with poor patient prognosis [J]. Cancer Res Clin Oncol, 2011, 137(4):557-566.

[30] Meng F, Henson R, Lang M, et al. Involvement of human 
micro-RNA in growth and response to chemotherapy in human cholangiocarcinoma cell lines [J]. Gastroenterology, 2006,130 (7):2113-2129.

[31] Si ML, Zhu S, Wu H, et al. MiR-21-mediated tumor growth [J]. Oncogene, 2007,26(19):2799-2803.

[32] Li Y, Li W, Yang Y, et al. MicroRNA-21 targets LRRFIP1 and contributes to VM-26 resistance in glioblastoma Multiforme [J] Brain Res, 2009,1286:13-18.

[33] Hwang JH, Voortman J, Giovannetti E, et al. Identification of microRNA-21 as a biomarker for chemoresistance and clinical outcome following adjuvant therapy in resectable pancreatic cancer [J]. PLoS One, 2010,5(5): e10630.
Submit your next manuscript to Chinese Journal of Cancer and take full advantage of:

- Open access

- No charge to authors

- Quickly published

- Thorough peer review

- Professionally edited

- No space constraints

- Indexed by PubMed, CA, and Google Scholar

Submit your manuscript at www.cjcsysu.com 